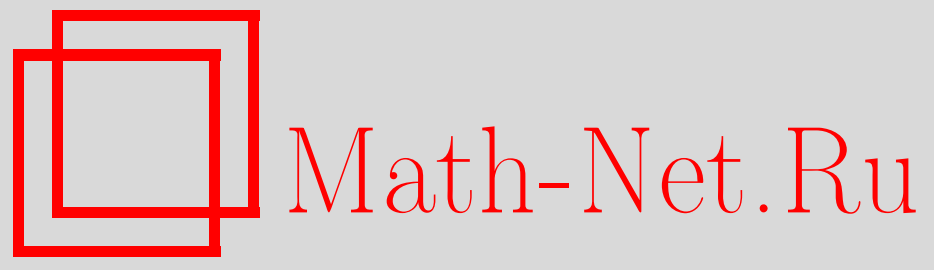

В. И. Трофимов, Об одном свойстве универсального метрического пространства Урысона, Матем. заметки, 2001, том 69, выпуск 2, 319-320

DOI: https://doi.org/10.4213/mzm685

Использование Общероссийского математического портала Math-Net.Ru подразумевает, что вы прочитали и согласны с пользовательским соглашением http://www.mathnet.ru/rus/agreement

Параметры загрузки:

IP : 54.147 .182 .235

26 апреля 2023 г., 12:53:40 


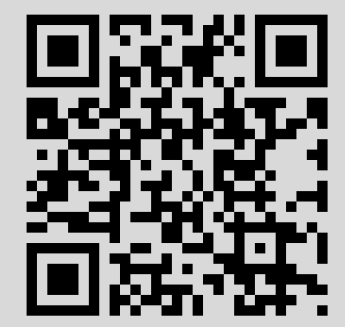




\section{ОБ ОДНОМ СВОЙСТВЕ УНИВЕРСАЛЬНОГО МЕТРИЧЕСКОГО ПРОСТРАНСТВА УРЫСОНА}

\section{В.И. Трофимов}

В работе [1] Урысоном построено замечательное метрическое пространство $U$, обладающее следуюшими свойствами:

1) $U$ - полное пространство со счетной базой;

2) $U$ содержит изометрический образ любого метрического пространства со счетной базой;

3) $U$ метрически однородно, т.е. для любых его конечных изометричных подпространств $A$ и $B$ существует изометрия пространства $U$, переводящая $A$ в $B$.

Согласно [1, теорема IV] метрическое пространство $U$ однозначно (с точностью до изометрии) определяется свойствами 1)-3).

Пусть $A$ и $B$ - подпространства метрического пространства $U$ и $\varphi$ - изометрия пространства $A$ на пространство $B$. Если $A$ и $B$ конечны, то согласно 2 ) существует такая изометрия $\psi$ пространства $U$, что $\psi(A)=B$. Заметим, что фактически в $[1$, с. $760-762]$ доказывается существование в этом случае даже изометрии $\psi$ пространства $U$ со свойством $\left.\psi\right|_{A}=\varphi$. В то же время, согласно теореме XII из [1] для бесконечных $A$ и $B$ изометрии $\psi$ пространства $U$ со свойством $\psi(A)=B$ может не существовать. Так (см. [1, с. 773$])$, если $S(x, \varepsilon)$ - сфера радиуса $\varepsilon>0$ с центром $x \in U$ метрического пространства $U$, то, с одной стороны, сушествует изометрия $\varphi^{\prime}$ подпространства $S(x, \varepsilon) \cup\{x\}$ метрического пространства $U$ на некоторое содержащееся в $S(x, \varepsilon)$ подпространство метрического пространства $U$, а с другой стороны, не существует изометрии пространства $U$, отображающей $S(x, \varepsilon)$ на собственное подмножество множества $S(x, \varepsilon)$. Следовательно, для изометричных подпространств $A=S(x, \varepsilon), B=\varphi^{\prime}(S(x, \varepsilon))$ метрического пространства $U$ не существует изометрии $\psi$ пространства $U$ со свойством $\psi(A)=B$. После завершения таким образом доказательства теоремы XII b [1, с. 773$]$ формулируется в качестве открытого следующий

Вопрос. Нельзя ли любые два конгруэнтных счетныx множества в $U$ перевести друг в друга изометрическим отображением пространства $U$ на себя?

(В принятой в [1] терминологии конгруэнтные множества в $U$ - это изометричные подпространства метрического пространства $U$.) Однако отрицательный ответ на сформулированный вопрос легко следует из доказанных в [1] результатов (что было анонсировано в [2]). Ниже приводятся соответствующие рассуждения.

Согласно [1, теорема VIII] существует такое счетное подпространство $C$ метрического пространства $U$, что $\bar{C}=S(x, \varepsilon)$ (здесь и далее используются введенные ранее обозначения). Содержащееся в $S(x, \varepsilon)$ подпространство $\varphi^{\prime}(C)$ метрического пространства $U$ изометрично $C$. Предположим, что существует изометрия $\psi^{\prime}$ пространства $U$, для которой $\psi^{\prime}(C) \subseteq \varphi^{\prime}(C)$. Тогда

$$
\psi^{\prime}(S(x, \varepsilon))=\psi^{\prime}(\bar{C}) \subseteq \overline{\psi^{\prime}(C)} \subseteq \overline{\varphi^{\prime}(C)} \subseteq S(x, \varepsilon)
$$

(С) В.И. Трофимов 
причем в силу $\varphi^{\prime}(x) \in S(x, \varepsilon)$ и $\rho\left(\varphi^{\prime}(x), \varphi^{\prime}(C)\right)=\rho(x, C)=\varepsilon$, где $\rho(\cdot, \cdot)$ - расстояние в $U$, имеем $\overline{\varphi^{\prime}(C)} \neq S(x, \varepsilon)$. Таким образом, изометрия $\psi^{\prime}$ пространства $U$ отображает $S(x, \varepsilon)$ на собственное подмножество множества $S(x, \varepsilon)$. Как было отмечено, последнее согласно $[1$, c. 773$]$ невозможно. Итак, для счетньх изометричных подпространств $C$ и $\varphi^{\prime}(C)$ метрического пространства $U$ не существует изометрии пространства $U$, отображающей $C$ в $\varphi^{\prime}(C)$.

Как было отмечено рецензентом, рассматриваемый выше вопрос из [1] отсутствует в работе [3], являющейся ранней версией [1]. Обе эти работы были отредактированы для печати П. С. Александровым уже без участия П. С. Урысона (подробности см. в $[1$, с. $776-777])$.

\section{СПИСОК ЦИТИРОВАННОЙ ЛИТЕРАТУРЫ}

1. Урысон П. С. // Труды по топологии и другим областям математики. Т. 2. М.: Гостехиздат, 1951. C. 747-777. 2. Trofimov V. I. // Paul Erdös and his Mathematics. Research Communications of the Conference Held in the Memory of Paul Erdös. Janos Bolyai Math. Soc.: Budapest, 1999. P. 255. 3. Urysohn P. S. // Bull. Sci. Math. 1927. V. 51. P. 43-64, 74-90.

Институт математики и механики УрО РАН, г. Екатеринбург

Поступило

E-mail: trofimov@imm.uran.ru

01.03.1999 